\title{
Highlights of mechanistic and therapeutic cachexia and sarcopenia research 2010 to 2012 and their relevance for cardiology
}

Markus S. Anker ${ }^{1}$, Stephan von Haehling ${ }^{2}$, Jochen Springer ${ }^{3}$, Maciej Banach ${ }^{4}$, Stefan D. Anker ${ }^{1,2}$

${ }^{1}$ Center for Clinical and Basic Research, IRCCS San Raffaele, Rome, Italy

2Applied Cachexia Research, Department of Cardiology, Charité,

Campus Virchow-Klinikum, Berlin, Germany

${ }^{3}$ Applied Cachexia Research, Center for Cardiovascular Research, Charité, Campus Mitte, Berlin, Germany

${ }^{4}$ Department of Hypertension, Medical University of Lodz, Poland

Submitted: 3 November 2012

Accepted: 6 November 2012

Arch Med Sci 2013; 9, 1: 166-171

DOI: 10.5114 /aoms.2013.33356

Copyright @ 2013 Termedia \& Banach

\begin{abstract}
Sarcopenia and cachexia are significant medical problems with a high diseaserelated burden in cardiovascular illness. Muscle wasting and weight loss are very frequent particularly in chronic heart failure and they relate to poor prognosis. Although clinically largely underestimated, the fields of cachexia and sarcopenia are of great relevance to cardiologists. In cachexia and sarcopenia a significant number of research publications related to basic science questions of muscle wasting and lipolysis were published between 2010 and 2012. Recently, the two processes of muscle wasting and lipolysis were found to be closely linked. Treatment research in pre-clinical models involves studies on a number of different therapeutic entities, including ghrelin, selective androgen receptor modulators (SARMs), as well as drugs targeting myostatin or melanocortin-4. In the human setting, studies using enobosarm (a SARM) and anamorelin (ghrelin) are in phase III. The last 3 years have seen significant efforts to define the field using consensus statements. In the future, these definitions should also be considered for guidelines and treatment trials in cardiovascular medicine. The current review aims to summarize important information and development in the fields of muscle wasting, sarcopenia and cachexia, focusing on findings in cardiovascular research, in order for cardiologists to have a better understanding of the progress in this still insufficiently known field.
\end{abstract}

Key words: cachexia, sarcopenia, muscle wasting, mechanism, therapy, cardiovascular illness, heart failure.

\section{Introduction}

The last decades have seen increasing interest in muscle wasting [1-5] and cachexia [6-16] research in chronic diseases and ageing. Indeed, both are significant medical problems with a high disease-related burden in terms of symptoms, quality of life and prognosis of patients [17-19] and with high socioeconomic expense [20]. Muscle wasting in general, sarcopenia, lipolysis and cachexia are very active research fields with great medical relevance [21-23]. New research is abundant and many comprehensive review articles have been written to cover the new findings on mechanisms and pathophysiology [24, 25] as well as symptoms [26, 27],

\section{Corresponding author:}

Stefan D. Anker MD, PhD

Applied Cachexia Research

Department of Cardiology

Charité Berlin

Campus Virchow-Klinikum

Augustenburger Platz 1

D-13353 Berlin, Germany

Phone: +4930450553506

Fax: +4930450553951

E-mail: s.anker@cachexia.de 
nutritional issues [28, 29], and new therapeutic options [30-33].

In this review we aimed to summarize the most important information on muscle wasting, sarcopenia and cachexia, focusing on findings in cardiovascular research, in order for cardiologists to have a better understanding of the progress in this still insufficiently known field. In order to reach a wide group of physicians and researchers, we have decided to publish this review simultaneously in two renowned journals (wide-scope and cardiologist ones).

\section{Search strategy}

We searched the electronic databases MEDLINE, EMBASE and SCOPUS (January 2010 to October 2012). Additionally, abstracts from national and international cardiovascular meetings were searched. The main search terms were: cachexia, sarcopenia, muscle wasting, mechanism, therapy, cardiovascular illness, and heart failure.

Muscle wasting is not only seen in chronically ill patients, but also prominent in acute and critical illness, including sepsis and septic shock [34], in hospitalized patients with acute and severe lung disease [35]. Patients with chronic heart failure were recently found to be affected by muscle wasting fulfilling the criteria of sarcopenia in $19.5 \%$ of a prospectively enrolled cohort of patients with stable disease [36]. Cachexia, on the other hand, is frequent in chronic kidney disease (CKD) [9, 37-39] and as part of the cardio-renal syndrome also affecting heart failure (HF) patients regularly [40-42]. Cachexia is also a significant problem in advanced heart disease itself [43] and related to comorbidities such as diabetes mellitus and chronic inflammation [44]. Abnormal cardiac parameters are seen in cachexia and may be associated with abnormal myocardial perfusion [45].

Much of the research on muscle wasting is focusing on regulators such myostatin [46], the ubiquitin-proteasome pathway [47], and free-radical production [48], but also on novel treatment approaches including the use of bile acids [49]. The latter has also been tested in humans with chronic heart failure (CHF) and showed improvements of endothelial function in arms and legs [50]. Limb anatomical changes may also cause peripheral neuropathy, which in turn has been shown to relate to poor quality of life [51]. Adaptive mechanisms in skeletal muscle are complex, involving the whole body, which develops anabolic/catabolic imbalance, anabolic failure and hypogonadism, as has been convincingly shown in CHF [52-54]. Other pathogenetic factors studied include sirtuin 1 , which has been suggested to be a relevant mechanistic factor in the development of insufficient regeneration of skeletal muscle in cancer cachexia
[55]. Desmin and tissue zinc redistribution are additional factors investigated for their role in muscle wasting $[56,57]$. Other important fields of research are related to metabolic syndrome and insulin resistance [58-61] as well as heat shock protein 72 [62] and muscle stem cell function [63] and related telomere alterations [64]. The role of genetic factors is less well understood at this stage, but related research efforts are underway [44].

Research on lipolysis [65] is a young but strongly evolving field. There is strong interaction between lipolysis and muscle wasting [66-68]. This may also be relevant in heart disease [69].

Biomarker research in the field of muscle wasting is active [70, 71]. Serum creatinine levels are understood to be an unspecific marker of muscle wasting [72], but recently a more specific marker (C-terminal agrin fragment) was described [73]. As a result of a consensus conference that took place in Toulouse in 2011, recently a consensus paper on blood analysis based as well as imaging based biomarkers defining sarcopenia was published [74]. Before such biomarkers can be considered clinically (outcome) validated, successful clinical intervention phase III trials are needed.

Interventional studies in the pre-clinical setting are frequently reported and include studies investigating insulin-like growth factor [75], ghrelin and ghrelin analogues [76, 77], as well as specific androgen receptor modulators (SARMs) [78], and myostatin antibodies [79]. In addition, pre-clinical models suggest that treatments to block the effects mediated via the melanocortin-4 receptor may be useful in cachexia and muscle wasting [80]. The melanocortin-4 receptor antagonist BL-6020/ 979 is orally available and ready for human testing $[17,81]$. The results so far are promising. Several other such compounds are in development [82]. Anabolic drugs are potentially of great relevance to $\mathrm{HF}$ where muscle wasting is highly prevalent [6]. Testosterone has already been tested and the results are promising [83].

In recent years, several interventional trials in humans have been performed, but for a number of them no final publications yet exist and we have to rely on meeting reports at conferences and press releases. Two promising drug treatment approaches are now in phase III clinical testing [84]. The SARM GTX-024 (enobosarm) [85] is being tested in two trials of 300 patients as to whether it can prevent or treat muscle wasting in non-small cell lung cancer. The two primary endpoints are changes in lean mass and changes in stair-climbing power [86]. The second trial program includes 2 trials of 477 patients each to investigate whether an orally available ghrelin (anamorelin) can positively impact on the disease progression of cachexia in non-small cell lung cancer [87]. The two primary 
endpoints are changes in lean mass and changes in hand-grip strength, which will also have relevance in future heart failure trials. Results for these clinical trial programs are expected to be available in 2014.

From a cardiological standpoint, it is interesting to note that even cardiovascular drugs are now in clinical testing in cachexia. MT-102 is an anabolic/catabolic transforming agent with $\beta 1$-blocking activity and it has direct anabolic and appetite stimulating effects in animal studies [88]. It is now being investigated in 132 patients with cachexia due to lung cancer or colorectal cancer [64]. Of interest, in the past also an angiotensin-converting enzyme inhibitor (imidapril) was investigated for use in cancer cachexia. Some promising results were obtained, but development programs were not completed due to lack of funding [89].

One treatment alone may not be sufficient to successfully treat sarcopenia or cachexia. Hence combination therapies have been proposed, including eicosapentaenoic acid and training exercise $[90,91]$, as well as anabolic steroids and exercise or more complex combinations such as megestrol acetate plus I-carnitine, celecoxib, and possibly also antioxidants [92, 93]. Nutritional studies have been a cornerstone of therapeutic research in cachexia and sarcopenia. Rozentryt et al. performed a clinical trial of nutritional intervention in cardiac cachexia [94]. A diet containing $600 \mathrm{kcal}$ per day for 6 weeks (with relatively high fat content to reduce volume) increased weight during the treatment and the subsequent 12-week follow-up period (70\% fat tissue, $30 \%$ muscle tissue) and had anti-inflammatory effects (reduction of TNF levels). This was accompanied by increases in cholesterol levels. Quality of life of patients improved for the 6-week period of the nutritional intervention, but decreased somewhat in the additional follow-up period.

Starting in 2008 [95], and with much more intensity in 2010 to 2012, a number of consensus-building activities to define cachexia and sarcopenia have been undertaken [96-99]. Also a more specific consensus document on the definition and stages of cancer cachexia has been published [100]. The reason for these activities is that it has been argued that treatments for cachexia (or sarcopenia) can only be developed when one can define it [101]. As much as this may be true, defining cachexia is not a simple task [102], and one can also postulate that at least as important as consensus definitions may in the long term be inclusion and exclusion criteria of trials per se. If they lead to regulatory treatment approval, then they will build the basis for approved educational initiatives that may likely develop more disease defining power than the consensus definitions ever could.
This may be similar to the situation regarding the definition of diastolic heart failure or HF with preserved ejection fraction [103, 104]. Guidelines and consensus papers will not be as powerful as the first successful therapies and the inclusion and exclusion criteria they used.

\section{Journal and conference activities}

Finally, the field of cachexia, sarcopenia and muscle wasting research is characterized by the development of several new scientific journals. In 2010, we founded the Journal of Cachexia, Sarcopenia and Muscle (JCSM). In 2011, the journal Skeletal Muscle, and in 2012 The Journal of Frailty and Aging (JFA) were founded. All these journals are free access or open access. Since 2000, six international cachexia conferences have taken place and reported extensively. Since 2000, the number of attendees has steadily increased from 150 to more than 400 in 2009 [105]. The next conference will take place from 8 to 11 December, 2013. Of even greater interest to those involved in treatment development in cachexia and muscle wasting in HF may be the 2013 Annual Congress of the Heart Failure Association of the European Society of Cardiology (Lisbon, 25-28 May, 2013), where on 28/29 May a trialist workshop on treatment development for cachexia and muscle wasting in heart failure and chronic obstructive pulmonary disease will be held.

\section{Acknowledgments}

Stephan von Haehling is consulting and has received honoraria for speaking from Professional Dietetics, Vifor, and Pfizer. Stefan D. Anker is consulting, and has received honoraria for speaking and/or attended advisory boards for Amgen Inc, Professional Dietetics, Psioxus Therapeutics, GTx, Helsinn, GSK, Sanofi, Regeneron, Novartis, Takeda, Servier, Chugai and Vifor. All other authors report no conflict of interest.

This paper is also published in parallel in International Journal of Cardiology.

\section{References}

1. Baumgartner RN, Koehler KM, Gallagher D, et al. Epidemiology of sarcopenia among the elderly in New Mexico. Am J Epidemiol 1998; 147: 755-63.

2. Janssen I, Heymsfield SB, Ross R. Low relative skeletal muscle mass (sarcopenia) in older persons is associated with functional impairment and physical disability. J Am Geriatr Soc 2002; 50: 889-96.

3. Janssen I. Influence of sarcopenia on the development of physical disability: the cardiovascular health study. J Am Geriatr Soc 2006; 54: 56-62.

4. Rolland Y, Lauwers-Cances V, Cournot M, et al. Sarcopenia, calf circumference, and physical function of elderly women: a cross-sectional study. J Am Geriatr Soc 2003; 51: $1120-4$

5. Lauretani F, Russo CR, Bandinelli S, et al. Age-associated changes in skeletal muscles and their effect on mobility: 
an operational diagnosis of sarcopenia. J Appl Physiol 2003; 95: 1851-60

6. Lees J. Incidence of weight loss in head and neck cancer patients on commencing radiotherapy treatment at a regional oncology centre. Eur J Cancer Care 1999; 8: 133-6.

7. Dewys WD, Begg C, Lavin PT, et al. Prognostic effect of weight loss prior to chemotherapy in cancer patients. Eastern Cooperative Oncology Group. Am J Med 1980; 69: 491-7.

8. Bachmann J, Heiligensetzer M, Krakowski-Roosen $\mathrm{H}$, Büchler MW, Friess H, Martignoni ME. Cachexia worsens prognosis in patients with resectable pancreatic cancer. J Gastrointest Surg 2008; 12: 1193-201.

9. Anker SD, Ponikowski P, Varney S, et al. Wasting as independent risk factor for mortality in chronic heart failure. Lancet 1997; 349: 1050-3.

10. Mak RH, Cheung W. Energy homeostasis and cachexia in chronic kidney disease. Pediatr Nephrol 2006; 21: 1807-14.

11. Koehler F, Doehner W, Hoernig S, Witt C, Anker SD, John M. Anorexia in chronic obstructive pulmonary disease - association to cachexia and hormonal derangement. Int J Cardiol 2007; 119: 83-9.

12. Vermeeren MA, Creutzberg EC, Schols AM, Postma DS, Pieters WR, Roldaan AC. COSMIC Study group. Prevalence of nutritional depletion in a large out-patient population of patients with COPD. Respir Med 2006; 100: 1349-55.

13. Wilson DO, Rogers RM, Wright EC, Anthonisen NR. Body weight in chronic obstructive pulmonary disease. The national institutes of health intermittent positivepressure breathing trial. Am Rev Respir Dis 1989; 139 . 1435-8.

14. Schols AM, Soeters PB, Dingemans AM, Mostert R, Frantzen PJ, Wouters EF. Prevalence and characteristics of nutritional depletion in patients with stable COPD eligible for pulmonary rehabilitation. Am Rev Respir Dis 1993; 147: 1151-6.

15. Elkan AC, Håkansson N, Frostegård J, Cederholm T, Hafström I. Rheumatoid cachexia is associated with dyslipidemia and low levels of atheroprotective natural antibodies against phosphorylcholine but not with dietary fat in patients with rheumatoid arthritis: a cross-sectional study. Arthritis Res Ther 2009; 11: R37.

16. Roubenoff R, Roubenoff RA, Ward LM, Holland SM, Hellmann DB. Rheumatoid cachexia: depletion of lean body mass in rheumatoid arthritis. Possible association with tumor necrosis factor. J Rheumatol 1992; 19: 1505-10.

17. von Haehling S, Morley JE, Anker SD. An overview of sarcopenia: facts and numbers on prevalence and clinical impact. J Cachexia Sarcopenia Muscle 2010; 1: 129-33.

18. von Haehling S, Anker SD. Cachexia as a major underestimated and unmet medical need: facts and numbers. J Cachexia Sarcopenia Muscle 2010; 1: 1-5.

19. Scherbakov N, Doehner W. Sarcopenia in stroke - facts and numbers on muscle loss accounting for disability after stroke. J Cachexia Sarcopenia Muscle 2011; 2: 5-8.

20. Scott D, Blizzard L, Fell J, Jones G. The epidemiology of sarcopenia in community living older adults: what role does lifestyle play? J Cachexia Sarcopenia Muscle 2011; 2: 125-34.

21. Dodson S, Baracos VE, Jatoi A, et al. Muscle wasting in cancer cachexia: clinical implications, diagnosis, and emerging treatment strategies. Annu Rev Med 2011; 62: 265-79.

22. von Haehling S, Lainscak M, Springer J, Anker SD. Cardiac cachexia: a systematic overview. Pharmacol Ther 2009; 121: 227-52.
23. Remels AH, Gosker HR, Langen RC, Schols AM. The mechanisms of cachexia underlying muscle dysfunction in COPD. J Appl Physiol 2012; DOI: 10.1152/japplphysiol. 00790.2012.

24. Lenk K, Schuler G, Adams V. Skeletal muscle wasting in cachexia and sarcopenia: molecular pathophysiology and impact of exercise training. I Cachexia Sarcopenia Muscle 2010; 1: 9-21.

25. Banach M, Rysz J. Current problems in hypertension and nephrology. Expert Opin Pharmacother 2010; 11: 2575-8.

26. Kilgour RD, Vigano A, Trutschnigg B, et al. Cancer-related fatigue: the impact of skeletal muscle mass and strength in patients with advanced cancer. J Cachexia Sarcopenia Muscle 2010; 1: 177-85.

27. Braun TP, Marks DL. Pathophysiology and treatment of inflammatory anorexia in chronic disease. J Cachexia Sarcopenia Muscle 2010; 1: 135-45.

28. Santarpia L, Contaldo F, Pasanisi F. Nutritional screening and early treatment of malnutrition in cancer patients. J Cachexia Sarcopenia Muscle 2011; 2: 27-35.

29. Dioguardi FS. Clinical use of amino acids as dietary supplement: pros and cons. J Cachexia Sarcopenia Muscle 2011; 2: 75-80.

30. Akamizu T, Kangawa K. Ghrelin for cachexia. J Cachexia Sarcopenia Muscle 2010; 1: 169-76.

31. Silvério R, Laviano A, Rossi Fanelli F, Seelaender M. I-Carnitine and cancer cachexia: clinical and experimental aspects. J Cachexia Sarcopenia Muscle 2011; 2: 37-44.

32. Dallmann R, Weyermann P, Anklin C, et al. The orally active melanocortin-4 receptor antagonist BL-6020/979: a promising candidate for the treatment of cancer cachexia. J Cachexia Sarcopenia Muscle 2011; 2: 163-74.

33. Trobec K, von Haehling S, Anker SD, Lainscak M. Growth hormone, insulin-like growth factor 1, and insulin signaling-a pharmacological target in body wasting and cachexia. J Cachexia Sarcopenia Muscle 2011; 2: 191-200.

34. Schefold JC, Bierbrauer J, Weber-Carstens S. Intensive care unit-acquired weakness (ICUAW) and muscle wasting in critically ill patients with severe sepsis and septic shock. J Cachexia Sarcopenia Muscle 2010; 1: 147-57.

35. Lainscak M, von Haehling S, Doehner W, et al. Body mass index and prognosis in patients hospitalized with acute exacerbation of chronic obstructive pulmonary disease. J Cachexia Sarcopenia Muscle 2011; 2: 81-6.

36. Fülster $S$, Tacke M, Sandek A, et al. Muscle wasting in patients with chronic heart failure: results from the Studies Investigating Co-morbidities Aggravating Heart Failure (SICA-HF). Eur Heart J 2012 (in press).

37. Mak RH, Ikizler AT, Kovesdy CP, Raj DS, Stenvinkel P, Kalantar-Zadeh K. Wasting in chronic kidney disease. J Cachexia Sarcopenia Muscle 2011; 2: 9-25.

38. Malyszko J, Bachorzewska-Gajewska H, Malyszko J, lainaLevin N, Kobus G, Dobrzycki S. Markers of kidney function in the elderly in relation to the new CKD-EPI formula for estimation of glomerular filtration rate. Arch Med Sci 2011; 7: 658-64.

39. Barylski M, Małyszko J, Rysz J, Myśliwiec M, Banach M. Lipids, blood pressure, kidney - what was new in 2011? Arch Med Sci 2011; 7: 1055-66.

40. Cicoira M, Anker SD, Ronco C. Cardio-renal cachexia syndromes (CRCS): pathophysiological foundations of a vicious pathological circle. J Cachexia Sarcopenia Muscle 2011; 2: 135-42.

41. Malyszko J, Zbroch E, Malyszko J, Mysliwiec M, Iaina A. The cardio-renal-anaemia syndrome predicts survival in peritoneally dialyzed patients. Arch Med Sci 2010; 6: 539-44. 
42. Couser WG, Riella MC. World Kidney Day 2011. Protect your kidneys, save your heart. Arch Med Sci 2011; 7: 1-4.

43. Kung T, Szabó T, Springer J, Doehner W, Anker SD, von Haehling S. Cachexia in heart disease: highlights from the ESC 2010. J Cachexia Sarcopenia Muscle 2011; 2: 63-9.

44. von Haehling S, Lainscak M, Doehner W, et al. Diabetes mellitus, cachexia and obesity in heart failure: rationale and design of the Studies Investigating Co-morbidities Aggravating Heart Failure (SICA-HF). J Cachexia Sarcopenia Muscle 2010; 1: 187-94.

45. Uematsu M, Akashi YJ, Ashikaga K, et al. Association between heart rate at rest and myocardial perfusion in patients with acute myocardial infarction undergoing cardiac rehabilitation - a pilot study. Arch Med Sci 2012; 8: 622-30.

46. Elkina Y, von Haehling S, Anker SD, Springer J. The role of myostatin in muscle wasting: an overview. J Cachexia Sarcopenia Muscle 2011; 2: 143-51.

47. Gielen S, Sandri M, Kozarez I, et al. Exercise training attenuates MuRF-1 expression in the skeletal muscle of patients with chronic heart failure independent of age: the randomized Leipzig Exercise Intervention in Chronic Heart Failure and Aging catabolism study. Circulation 2012; 125: 2716-27.

48. Sullivan-Gunn MJ, Campbell-O'Sullivan SP, Tisdale MJ, Lewandowski PA. Decreased NADPH oxidase expression and antioxidant activity in cachectic skeletal muscle. J Cachexia Sarcopenia Muscle 2011; 2: 181-8.

49. Tschirner A, von Haehling S, Palus S, Doehner W, Anker SD, Springer J. Ursodeoxycholic acid treatment in a rat model of cancer cachexia. J Cachexia Sarcopenia Muscle 2012; 3: 31-6.

50. von Haehling S, Schefold JC, Jankowska EA, et al. Ursodeoxycholic acid in patients with chronic heart failure: a double-blind, randomized, placebo-controlled, crossover trial. J Am Coll Cardiol 2012; 59: 585-92.

51. Ulubay G, Ulasli SS, Bozbas SS, Ozdemirel T, Karatas M. Effects of peripheral neuropathy on exercise capacity and quality of life in patients with chronic obstructive pulmonary diseases. Arch Med Sci 2012; 8: 296-302.

52. Anker SD, Chua TP, Ponikowski P, et al. Hormonal changes and catabolic/anabolic imbalance in chronic heart failure and their importance for cardiac cachexia. Circulation 1997; 96: 526-34.

53. Jankowska EA, Filippatos G, Ponikowska B, et al. Reduction in circulating testosterone relates to exercise capacity in men with chronic heart failure. J Card Fail 2009; 15: 442-50.

54. Jankowska EA, Biel B, Majda J, et al. Anabolic deficiency in men with chronic heart failure: prevalence and detrimental impact on survival. Circulation 2006; 114: 1829-37.

55. Toledo M, Busquets S, Ametller E, López-Soriano FJ, Argilés JM. Sirtuin 1 in skeletal muscle of cachectic tumourbearing rats: a role in impaired regeneration? J Cachexia Sarcopenia Muscle 2011; 2: 57-62.

56. Russ DW, Grandy JS. Increased desmin expression in hindlimb muscles of aging rats. J Cachexia Sarcopenia Muscle 2011; 2: 175-80.

57. Siren PM, Siren MJ. Systemic zinc redistribution and dyshomeostasis in cancer cachexia. J Cachexia Sarcopenia Muscle 2010; 1: 23-33.

58. Seneff S, Wainwright G, Mascitelli L. Is the metabolic syndrome caused by a high fructose, and relatively low fat, low cholesterol diet? Arch Med Sci 2011; 7: 8-20.
59. Abbatecola AM, Paolisso G, Fattoretti P, et al. Discovering pathways of sarcopenia in older adults: a role for insulin resistance on mitochondria dysfunction. J Nutr Health Aging 2011; 15: 890-5.

60. Gluba A, Mikhailidis DP, Lip GY, Hannam S, Rysz J, Banach $M$. Metabolic syndrome and renal disease. Int J Cardiol 2012 (in press).

61. Athyros VG, Giouleme O, Ganotakis ES, et al. Safety and impact on cardiovascular events of long-term multifactorial treatment in patients with metabolic syndrome and abnormal liver function tests: a post hoc analysis of the randomised ATTEMPT study. Arch Med Sci 2011; 7: 796-805.

62. Gehrig SM, van der Poel C, Sayer TA, et al. Hsp72 preserves muscle function and slows progression of severe muscular dystrophy. Nature 2012; 484: 394-8.

63. Gilbert PM, Havenstrite KL, Magnusson KE, et al. Substrate elasticity regulates skeletal muscle stem cell self-renewal in culture. Science 2010; 329: 1078-81.

64. Sacco A, Mourkioti F, Tran R, et al. Short telomeres and stem cell exhaustion model Duchenne muscular dystrophy in mdx/mTR mice. Cell 2010; 143: 1059-71.

65. Stephens NA, Skipworth RJ, Macdonald AJ, Greig CA, Ross JA, Fearon KC. Intramyocellular lipid droplets increase with progression of cachexia in cancer patients. J Cachexia Sarcopenia Muscle 2011; 2: 111-7.

66. Das SK, Eder S, Schauer S, et al. Adipose triglyceride lipase contributes to cancer-associated cachexia. Science 2011; 333: 233-8.

67. Arner P. Medicine. Lipases in cachexia. Science 2011; 333 163-4.

68. Fearon KC. Cancer cachexia and fat-muscle physiology. N Engl J Med 2011; 365: 565-7.

69. Pajak B, Orzechowska S, Pijet B, et al. Crossroads of cytokine signaling: the chase to stop muscle cachexia J Physiol Pharmacol 2008; 59 (Suppl 9): 251-64.

70. Chen F, Lam R, Shaywitz D, et al. Evaluation of early biomarkers of muscle anabolic response to testosterone. J Cachexia Sarcopenia Muscle 2011; 2: 45-56.

71. Gluba A, Bielecka A, Mikhailidis DP, et al. An update on biomarkers of heart failure in hypertensive patients. J Hypertens 2012; 30: 1681-9.

72. Patel SS, Molnar MZ, Tayek JA, et al. Serum creatinine as a marker of muscle mass in chronic kidney disease: results of a cross-sectional study and review of literature. J Cachexia Sarcopenia Muscle 2012 in press, DOI: 10.1007/s13539-012-0079-1.

73. Hettwer S, Dahinden P, Kucsera S, et al. Elevated levels of a C-terminal agrin fragment identifies a new subset of sarcopenia patients. Exp Gerontol 2012 in press, DOI: 10.1016/j.exger.2012.03.002.

74. Cesari $M$, Fielding RA, Pahor $M$, et al.; International Working Group on Sarcopenia. Biomarkers of sarcopenia in clinical trials-recommendations from the International Working Group on Sarcopenia. J Cachexia Sarcopenia Muscle 2012; 3: 181-90.

75. Schmidt K, von Haehling S, Doehner W, Palus S, Anker SD, Springer J. IGF-1 treatment reduces weight loss and improves outcome in a rat model of cancer cachexia. I Cachexia Sarcopenia Muscle 2011; 2: 105-9.

76. Müller TD, Perez-Tilve D, Tong J, Pfluger PT, Tschöp MH. Ghrelin and its potential in the treatment of eating/ wasting disorders and cachexia. J Cachexia Sarcopenia Muscle 2010; 1: 159-67.

77. Stepien M, Rosniak-Bak K, Paradowski M, et al. Waist circumference, ghrelin and selected adipose tissue-derived adipokines as predictors of insulin resistance in obese 
patients: preliminary results. Med Sci Monit 2011; 17: PR13-8.

78. Yarrow JF, McCoy SC, Borst SE. Tissue selectivity and potential clinical applications of trenbolone (17betahydroxyestra-4,9,11-trien-3-one): a potent anabolic steroid with reduced androgenic and estrogenic activity. Steroids 2010; 75: 377-89.

79. Zhou X, Wang JL, Lu J, et al. Reversal of cancer cachexia and muscle wasting by ActRIIB antagonism leads to prolonged survival. Cell 2010; 142: 531-43.

80. Peter JC, Rossez H, Weckering M, et al. Protective effects of an anti-melanocortin-4 receptor scFv derivative in lipopolysaccharide-induced cachexia in rats. J Cachexia Sarcopenia Muscle 2012 [Epub ahead of print], PMID: 22911214.

81. von Haehling S, Stepney R, Anker SD. Advances in understanding and treating cardiac cachexia: highlights from the 5th Cachexia Conference. Int J Cardiol 2010; 144: 347-9.

82. Scarlett JM, Bowe DD, Zhu X, Batra AK, Grant WF, Marks DL. Genetic and pharmacologic blockade of central melanocortin signaling attenuates cardiac cachexia in rodent models of heart failure. J Endocrinol 2010; 206: 121-30.

83. Malkin CJ, Channer KS, Jones TH. Testosterone and heart failure. Curr Opin Endocrinol Diabetes Obes 2010; 17: 262-8.

84. Thum T, Springer J. Breakthrough in cachexia treatment through a novel selective androgen receptor modulator? J Cachexia Sarcopenia Muscle 2011; 2: 121-3.

85. Dalton JT, Barnette KG, Bohl CE, et al. The selective androgen receptor modulator GTX-024 (enobosarm) improves lean body mass and physical function in healthy elderly men and postmenopausal women: results of a double-blind, placebo-controlled phase II trial. J Cachexia Sarcopenia Muscle 2011; 2: 153-61.

86. http://www.clinicaltrials.gov - NCT01355497 \& NCT01355484.

87. http://www.clinicaltrials.gov - NCT01387269 \& NCT01387282.

88. Stewart Coats AJ, Srinivasan V, Surendran J, et al.; the ACT-ONE Trial Investigators. The ACT-ONE trial, a multicentre, randomised, double-blind, placebo-controlled, dose-finding study of the anabolic/catabolic transforming agent, MT-102 in subjects with cachexia related to stage III and IV non-small cell lung cancer and colorectal cancer: study design. J Cachexia Sarcopenia Muscle 2011; 2: 201-7.

89. Schanze N, Springer J. Evidence for an effect of ACE inhibitors on cancer cachexia. J Cachexia Sarcopenia Muscle 2012; 3: 139.

90. Penna F, Busquets S, Pin F, et al. Combined approach to counteract experimental cancer cachexia: eicosapentaenoic acid and training exercise. I Cachexia Sarcopenia Muscle 2011; 2: 95-104.

91. von Haehling S. The muscle in dire straits: mechanisms of wasting in heart failure. Circulation 2012; 125: 2686-8.

92. Macci? A, Madeddu C, Gramignano G, et al. A randomized phase III clinical trial of a combined treatment for cachexia in patients with gynecological cancers: evaluating the impact on metabolic and inflammatory profiles and quality of life. Gynecol Oncol 2012; 124: 417-25.

93. Madeddu C, Dessí M, Panzone F, et al. Randomized phase III clinical trial of a combined treatment with carnitine + celecoxib \pm megestrol acetate for patients with cancer- related anorexia/cachexia syndrome. Clin Nutr 2012; 31: 176-82.

94. Rozentryt $\mathrm{P}$, von Haehling $\mathrm{S}$, Lainscak $\mathrm{M}$, et al. The effects of a high-caloric protein-rich oral nutritional supplement in patients with chronic heart failure and cachexia on quality of life, body composition, and inflammation markers: a randomized, double-blind pilot study. J Cachexia Sarcopenia Muscle 2010; 1: 35-42.

95. Evans WJ, Morley JE, Argilés J, et al. Cachexia: a new definition. Clin Nutr 2008; 27: 793-9.

96. Fielding RA, Vellas B, Evans WJ, et al. Sarcopenia: an undiagnosed condition in older adults. Current consensus definition: prevalence, etiology, and consequences. International working group on sarcopenia. J Am Med Dir Assoc 2011; 12: 249-56.

97. Morley JE, Abbatecola AM, Argiles JM, et al.; Society on Sarcopenia, Cachexia and Wasting Disorders Trialist Workshop. Sarcopenia with limited mobility: an international consensus. J Am Med Dir Assoc 2011; 12: 403-9.

98. Argilés JM, López-Soriano FJ, Toledo M, Betancourt A, Serpe R, Busquets S. The cachexia score (CASCO): a new tool for staging cachectic cancer patients. J Cachexia Sarcopenia Muscle 2011; 2: 87-93.

99. Muscaritoli M, Anker SD, Argilés J, et al. Consensus definition of sarcopenia, cachexia and pre-cachexia: joint document elaborated by Special Interest Groups (SIG) "cachexia-anorexia in chronic wasting diseases" and "nutrition in geriatrics". Clin Nutr 2010; 29: 154-9.

100. Fearon K, Strasser F, Anker SD, et al. Definition and classification of cancer cachexia: an international consensus. Lancet Oncol 2011; 12: 489-95.

101. Fearon K, Evans WJ, Anker SD. Myopenia-a new universal term for muscle wasting. J Cachexia Sarcopenia Muscle 2011; 2: 1-3.

102. Baracos VE. Pitfalls in defining and quantifying cachexia. J Cachexia Sarcopenia Muscle 2011; 2: 71-3.

103. Alagiakrishnan K, Banach M, Jones LG, Datta S, Ahmed A, Aronow WS. Update on diastolic heart failure or heart failure with preserved ejection fraction in the older adults. Ann Med 2012 in press, DOI: 10.3109/ 07853890. 2012.660493.

104. Karasek D, Sinkiewicz W, Błażejewski J. Relationship between B-type natriuretic peptide serum level, echocardiographic TEI index and the degree of diastolic dysfunction in patients with heart failure with preserved systolic function. Arch Med Sci 2011; 7: 449-56.

105. von Haehling S, Anker SD. The 6th Cachexia Conference: an introduction to clinical and basic research in an exiting area. J Cachexia Sarcopenia Muscle 2011; 2: 189-90. 